\section{Acute abdomen after allogenic hematopoietic stem cell transplantation}

\author{
Alessandro Crocoli,1 Daria Pagliara,2 \\ Franco Locatelli,2 Alessandro Inserra ${ }^{1}$ \\ 1Department Of Surgery and \\ 2Department of Hematology and \\ Oncology, Pediatric Hospital Bambino \\ Gesù, Rome, Italy
}

\section{Abstract}

We report a case of a patient who underwent allogenic hematopoietic stem cell transplantation complicated by acute colonic pseudo obstruction who required surgery after failure of conservative therapy.

\section{Case Report}

A 14-year old Caucasian male was admitted to our Department of Pediatric HematologyOncology with a diagnosis of acute lymphoid leukemia (ALL Pro-B/CALL) in the absence of cytogenetic abnormalities. He received chemotherapy according to the AIEOP LLA 2006 protocol in the high-risk group because of high levels of minimal residual disease (MRD). The patient was programmed to undergo allogeneic hematopoietic stem cell transplantation (allo-HSCT) from a matched unrelated donor. MRD level, evaluated before starting the conditioning regimen, was negative. The patient underwent myeloablative conditioning regimen based on total body irradiation, thiotepa, fludarabine and ATG. Graft-versus-host disease prophylaxis consisted of administration of cyclosporine beginning on Day -2 before allo-HSCT and methotrexate (on Days $+1,+3,+6$ and +11 after allo-HSCT).

On Day +9 the patient started suffering from diffuse abdominal, watery diarrhea, vomiting and fever. Laboratory findings showed increased C-reactive protein (CRP, $9.96 \mathrm{mg} / \mathrm{dl}$ ) with absolute neutrophil count of $0.01 \times 109 / \mathrm{L}$. Broad spectrum antibiotic therapy was started. During the following days, his clinical condition progressively deteriorated; abdominal X-ray showed a large bowel distension, while ultrasonography showed ascites. Evaluation of the viral load for adenovirus showed a high level of virus copies in the blood $(296,0000$ copies/mL) while cultures obtained from the central venous catheter (CVC) and from peripheral blood revealed a multiresistant Klebsiella Pneumoniae colo- nization. The CVC was removed and tip culture confirmed the presence of $K$. Pneumonia. Oliguria was present on day +20 with evidence of increased serum creatinine level $(3.26 \mathrm{mg} / \mathrm{dL})$. The patient also started suffering from multiple episodes of hematemesis and melena. There was a further aggravation of his general condition on day +23 with persistent hematic stagnation from the nasogastric tube, absence of both stool and air emission with radiological evidence of bowel obstruction, as well as presence of acute renal failure. Given his hemodynamic instability, the patient was transferred to the intensive care unit. Peritoneal drainage was put in place but abdominal distension did not respond to conservative therapies. An explorative laparotomy was, therefore, planned: gastrostomy with removal of large gastric clot occupying the whole stomach, hemodialysis central venous catheter placement were performed. No intestinal diversion was performed both for the rapid worsening of his hemodynamic condition and for the high risk of bacterial superinfection. Despite continuous support with vasoactive drugs, the continuous veno-venous hemofiltration and the antibiotical shift, the patient died on the $2^{\text {nd }}$ day after surgery.

Abdominal complications in patients undergoing allo-HSCT could lead to catastrophic events, especially in the pediatric population. ${ }^{1}$ Use of myeloablative therapies with subsequent immunodeficiency, together with underlying diseases and related treatments, may result in life-threatening situations which can prove fatal. In this respect, acute colonic pseudo obstruction (ACPO) represents a rare condition which has been reported in critical patients with prolonged hospitalization who develop neutropenic enterocolitis as a consequence of intensive therapies, such as antibiotics, chemotherapy and radiation. ${ }^{2}$ Bowel mucosal damage, induced by such treatments, determines bacterial superinfection with intestinal wall colonization. The mechanism of subsequent bowel paralysis is still unclear, although many Authors have speculated about the role of autonomic nervous dysfunction induced by wall injury. 2,3 As a consequence, progressive dilation both of the large and small bowel, with clinical and radiological evidence of ileus, leads to complete intestinal obstruction in the absence of any mechanical blockage. Management of ACPO should be initially conservative, with treatment of underlying disease, administration of broad spectrum antibiotics, reduction/interruption of analgesic drugs, bowel rest with interruption of oral feeding, total parenteral nutrition, continuous nasogastric aspiration and administration of drugs such as neostigmine with a direct effect on the cholinergic activity of the
Correspondence: Alessandro Crocoli, Department Of Surgery, Pediatric Hospital Bambino Gesù, Piazza S.Onofrio 4, 00165 Rome, Italy.

Tel. 06.68592030 - Fax: 06.68592012.

E-mail: alessandro.crocoli@opbg.net

Key words: acute abdomen, acute lymphoid leukemia, allogenic hematopoietic stem cell transplantation

Contributions: AC, AI wrote the report and performed surgery; DP, FL treated the patient over the whole course of the disease.

Conflict of interest: the authors report no conflicts of interest.

Received for publication: 2 October 2011.

Revision received: 24 November 2011.

Accepted for publication: 25 November 2011.

This work is licensed under a Creative Commons Attribution NonCommercial 3.0 License (CC BYNC 3.0).

(C)Copyright A. Crocoli et al., 2011

Licensee PAGEPress, Italy

Pediatric Reports 2011; 3:e32

doi:10.4081/pr.2011.e32

bowel wall. ${ }^{2-4}$ Surgery must only be considered for patients who do not respond to conservative management and for those who have complications, such as intestinal ischemia/perforation. Moreover, immunocompromised children with ACPO, during the acute phase of disease, are not able to tolerate either surgery or the sequelae of procedures performed to solve the bowel distention, such as temporary ostomy or decompressive laparotomy with delayed closure. ${ }^{1-4}$ In our patient, the ACPO arose during the aplastic phase after allo-HSCT as a consequence of neutropenic enterocolitis. This condition was also associated with dissemination of the adenovirus infection, the multiresistant $\mathrm{K}$. Pneumoniae sepsis and the development of acute renal failure with uremic syndrome. Subsequent oligo-anuria and hemorrhagic uremic gastritis determined a rapid worsening of his general condition, with a direct impact on abdominal symptoms. The decision not to perform decompressive ostomy was made according to the elevated risk of bacterial contamination and superinfection during and after surgery.

Despite the poor outcome, in children with onco-hematologic disease who develop ACPO during the course of treatment, surgery should only be performed in selected cases which do not respond to conservative therapies, this being considered salvage therapy. ${ }^{3,4}$ In these patients, to reduce the risk of fatal complications, surgeons must evaluate the need for minimal surgery to facilitate relief of 
the bowel distention. The timing and type of procedure should be carefully evaluated according to patient condition. In the case of intestinal pneumatosis on abdominal X-ray, there should be prompt surgical intervention in order to reduce the risk of perforation with consequent peritoneal contamination and increased risk of septic shock. In this respect, intestinal diversion with temporary/definitive ostomy is the first-line treatment, since primary anastomosis after resection is contraindicated because of the high risk of dehiscence, especially in this pediatric population.

\section{References}

1. Dauplaise DJ, Barnett SJ, Frischer JS, et al. Decompressive abdominal laparotomy for abdominal compartment syndrome in an unengrafted bone marrow recipient with septic shock. Crit Care Res Pract 2010; PMID 20948887.

2. Breccia M, Girmenia C, Mecarocci S, et al. Ogilvie's syndrome in acute myeloid leukemia: pharmacological approach with neostigmine. Ann Hematol 2001;80:614-6.
3. Lee JW, Bang KW, Jang PS, et al. Neostigmine for the treatment of acute colonic pseudo-obstruction (ACPO) in pediatric hematologic malignancies. Korean J Hematol 2010;45:62-5.

4. Kumar A, Saltzman D, Shukula M, et al. Diagnostic peritoneal lavage for assessing acute abdomen in pediatric oncology and stem cell transplantation patients. J Pediatr Hematol Oncol 2004;26:824-6. 